\title{
Conf- 9709121
}

\section{ISOTROPIZATION OF NEMATIC LIQUID CRYSTALS BY TMDSC}

Wei Chen. Mark Dadmun. Ge Zhang. Andreas Boller and Bernhard Wunderlich

Dept. of Chemistry. The Lniversity of Tennessee. Knoxville. TN 37996-16097 Chem. and Anal. Sci. Div.. Oak Ridge Natl. Lab.. Oak Ridge. TN 37831-619

\section{ECENG}

\section{Abstract}

Temperature-modulated differential scanning calorimetry (TMDSC) and traditional DSC are used to study the transition between the nematic liquid crystalline state and the isotropic liquid for two small molecules [4.4'-azoxyanisole and N.N'-bis(4-noctyloxybenzal)-1.4-phenylenediamine] and one macromolecule (4,4'-dihydroxy- $\alpha$ methylstilbene copolymerized with a 1:1 molar mixture of 1.7-dibromoheptane and 1.9-dibromononane). The DSC measurements with 4.4'-azoxyanisole were used for temperature calibration with varying heating and cooling rates. Quasi-isothermal TMDSC with small temperature amplitude and standard TMDSC with underlying heating and cooling rates were utilized to analyze the breadth of the transitions. It could be verified that the isotropization transition of a nematic liquid crystal is, indeed. reversible for all three molecules. The nature of the transition changes. however. from relatively sharp. for smail. rigid molecules. to about three kelvins wide for the small molecule with flexible ends. to as broad as $20 \mathrm{~K}$ for the macromolecule. It was also demonstrated that quantitative heats of fusion of sharp transitions can be extracted from TMDSC. but only from the time-domain heat-flow signal.

\section{Introduction}

The transition from the nematic ( $\mathrm{N}$ ) liquid crystalline phase (LC) to the isotropic (I) melt is generally assumed to be a first-order. equilibrium transition [1]. In other words. the transition occurs at the same temperature on cooling and heating. and no nucleation is required for the $I \rightarrow N$ transition. in contrast to crystallization. Because of this reversibility. it has been demonstrated for the Perkin-Elmer power compensation DSC that the $\mathrm{N} \rightarrow$ I transition can be used as standard for temperature calibration on heating and $\mathrm{I} \rightarrow \mathrm{N}$ for cooling [2.3]. In this paper a first investigation of these transitions is described using a heat-flux type, temperature-modulated differential scanning calorimeter (TMDSC). The transition is followed through the entire temperature range of the transition by using traditional DSC. quasi-isothermal TMDSC. and standard TMDSC with an underlying heating rate $\langle q\rangle$. It will be shown that the transition from a nematic LC to the isotropic melt is. indeed. an equilibrium transition without need of nucleation. Dependent on the molecular character of the sample. the temperature range of the transition varies. however. from very narrow. to as much as $20 \mathrm{~K}$. i.e. the nematic to isotropic transition has an intrinsic breadth. The selection of samples for temperature calibration becomes. thus. crucial since the width of the transition may cause confusion in data interpretation. 


\section{DISCLAMERR}

Portions of this docoment may be illegible in electronic image prodnets. Images are produced from the best available original document 


\section{DISCLAIMER}

This report was prepared as an account of work sponsored by an agency of the United States Government. Neither the United States Government nor any agency thereof, nor any of their employees, make any warranty, express or implied, or assumes any legal liability or responsibility for the accuracy, completeness, or usefulness of any information, apparatus, product, or process disclosed, or represents that its use would not infringe privately owned rights. Reference herein to any specific commercial product, process, or service by trade name, trademark, manufacturer, or otherwise does not necessarily constitute or imply its endorsement, recommendation, or favoring by the United States Government or any agency thereof. The views and opinions of authors expressed herein do not necessarily state or reflect those of the United States Government or any agency thereof. 
In previous papers we have explored the advantages and limits of TMDSC [4-8]. Brietly, to modulate DSC. one adds a sinusoidal oscillation to the block temperature $T_{h}$ that would have otherwise increased linearly for traditional DSC:

$$
T_{h}=T_{0}+\langle q\rangle t-A_{T_{h}} \sin (\omega t)
$$

where $T_{v}$ is the temperature at time $t=0 ;\langle q\rangle$, the underlying scanning rate: $A_{T_{b}}$, the amplitude of modulation at the block temperature: and $\omega$. the modulation frequency in radians. The steady state temperature of the sample calorimeter is:

$$
T_{\mathrm{s}}=\mathrm{T}_{0}-\langle\mathrm{q}\rangle \mathrm{t}-\frac{\mathrm{q}>\mathrm{C}_{\mathrm{c}}}{\mathrm{k}}+\mathrm{A} \sin (\omega \mathrm{t}-\varepsilon)
$$

where $A$ is the maximum modulation amplitude of $T_{s}$, fixed as a run parameter. and $\varepsilon$ is the phase lag between the reference oscillation and the sample temperature. During isotropization of a liquid crystal. the latent heat is very small. therefore. the calorimeter is expected to stay close to steady-state (depending on the sharpness of the transition). Full descriptions of traditional DSC. quasi-isothermal TMDSC and standard TMDSC are given in refs. [8.9]. [4.8], and [5.6.8], respectively.

\section{Experimental}

A commercial TMDSC. the Thermal Analyst 2910 system with liquid-nitrogen cooling-accessory ( $\mathrm{LNCA}$ ) from TA Instruments Inc. (MDSC ${ }^{\mathrm{TM}}$ ), was used in this study: Dry nitrogen gas with a flow rate of $20 \mathrm{~mL} / \mathrm{min}$ was purged through the DSC cell. The temperature of the TMDSC equipment was initially calibrated in the standard DSC mode at $10 \mathrm{~K}$ min ' by using the transition peaks for cyclohexane $(186.09$ and $297.7 \mathrm{~K})$. octane $(216.15 \mathrm{~K})$, water $(273.15 \mathrm{~K})$, and indium $(429.75 \mathrm{~K})$. The transition temperatures of the standards are chosen at the extrapolated onsets [9]. Following the same procedure, the isotropization temperatures of the liquid crystals under investigation are determined on heating and cooling. The heat flow was calibrated with the heat of fusion of indium $\left(28.45 \mathrm{Jg}{ }^{\prime}\right)$.

In this work. the following experiments were performed: (1) The transition temperatures were measured with traditional DSC as a function of scanning rate (from $=0.2$ to $\pm 20.0 \mathrm{~K} \mathrm{~min}^{\prime}$ by the onset of isotropization and ordering). (2) Quasiisothermal TMDSC was carried out with a modulation amplitude $A=0.1 \mathrm{~K}$. coupled with a modulation period $\mathrm{p}=60 \mathrm{~s}$, and step-wise temperature-increments of $0.2 \mathrm{~K}$. Except for cases specified. the quasi-isothermal runs lasted 20 minutes. each. The last 10 minutes were used for data collection. (3) Standard TMDSC runs were performed with underlying heating rates $\langle\mathrm{q}\rangle= \pm 0.2 \mathrm{~K} \mathrm{~min}{ }^{1}$ and modulation parameters $p=60 \mathrm{~s}, \mathrm{~A}=0.5 \mathrm{~K}$. With these parameters the maximum heating and cooling rates $q=d T / d t$ are 3.3 and $2.9 \mathrm{~K} \mathrm{~min}^{-1}$. as calculated from Eq. (2).

The liquid crystal materials chosen for this study were: (1) 4,4'-azoxyanisole. obtained from Aldrich Chemical Co. (impurities $<2 \%$ ). It is a typical, small-molecule. nematic LC without major flexible appendages. The transition crystal $\rightarrow \mathrm{N}$ occurs at $T_{d}=391 \mathrm{~K}$ (disordering), $\mathrm{N} \rightarrow \mathrm{I}$ at $\mathrm{T}_{\mathrm{i}}=408 \mathrm{~K} . \Delta \mathrm{H}=2.56 \mathrm{~J} \mathrm{~g}{ }^{\prime}$. (2) N.N'-bis(4-n- 
uctyloxybenzal)-1.t-phenylenediamine (abbreviated as OOBPD). It was synthesized following the method of ref. [10]. This sample shows multiple transitions on heating, characterized fully earlier [11.12]. In this study, attention will be focused on the isotropization at $T=501 \mathrm{~K}$. This sample has flexible octyloxy-groups attached to its rigid center. the mesogen. (3) A main-chain. macromolecular LC-forming polyether was synthesized by coupling 4.4'-dihydroxy- $\alpha$-methylstilbene (DHMS) with a $1: 1$ molar mixture of 1.7-dibromoheptane and 1.9-dibromononane. The name of this random copolymer is abbreviated as DHMS-7.9. The molar mass of DHMS-7.9 is $36.000 \mathrm{Da}$. It forms a nematic phase between $400 \mathrm{~K}$ and $470 \mathrm{~K}[13,14]$.

\section{Results}

Figure 1 shows the onset temperatures for the isotropization and ordering of 4,4'azoxyanisole as a function of scanning rates on both. heating and cooling (solid circles) measured by traditional DSC. The transition temperatures change linearly

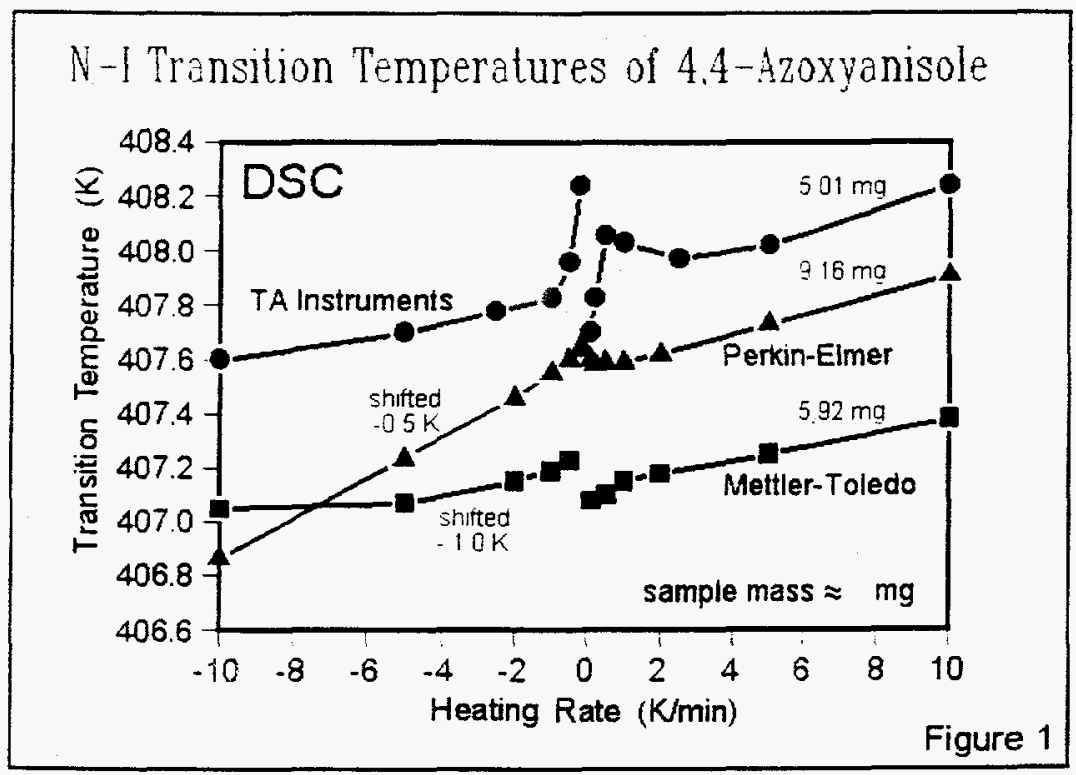

with heating/cooling rates. A linear equation can be used to represent the temperature dependence of the onset temperatures with heating rate q (data represented by the upper curve. solid circles):

$$
\mathrm{T}_{\mathrm{i}}-0.030 \times \mathrm{q}+407.93
$$

The equilibrium $T$, of 4.4'-Azoxyanisole is, thus. $407.93 \mathrm{~K}(\mathrm{q}=0)$.

It is of interest to note that a systematic deviation from the linear dependence of $T$, occurs as $q$ approaches zero. This deviation is considered to be an instrument effect. To elucidate this effect. the experiments were repeated on the different DSCs of Perkin-Elmer (solid triangles) and Mettler-Toledo DSC (solid squares). For clarity, these two data sets are shifted by 0.5 and $1.0 \mathrm{~K}$ to lower temperature. 


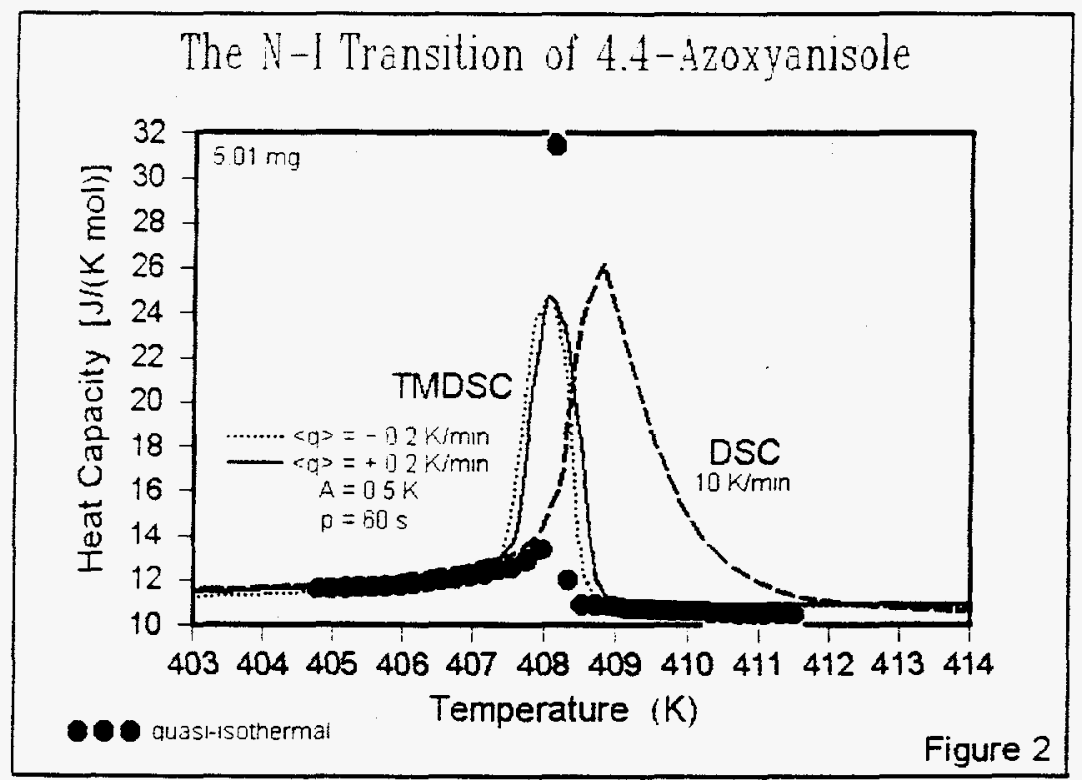

Plots of the $\mathrm{N} \rightarrow$ I transitions of 4.4'-Azoxyanisole. OOBPD and DHMS-7.9 measured under different DSC modes are illustrated in Figs. 2-4. respectively. The dashed lines represent the traditional DSC measurements with a heating rate of 10 $\mathrm{K}$ min '. The solid and dotted lines represent the standard TMDSC traces with an underlying heating and cooling rate. respectively. The solid circles mark the quasiisothermal TMDSC runs. For DHMS-7.9. the quasi-isothermal TMDSC was performed with runs of $15 \mathrm{~min}$ (circles) and $30 \mathrm{~min}$ (squares) at each temperature.

The onset temperatures for the 4.4'-Azoxyanisole by traditional DSC depend on scanning rate. as shown in Fig. 1 and by Eq. (3). The cause of this shift is the

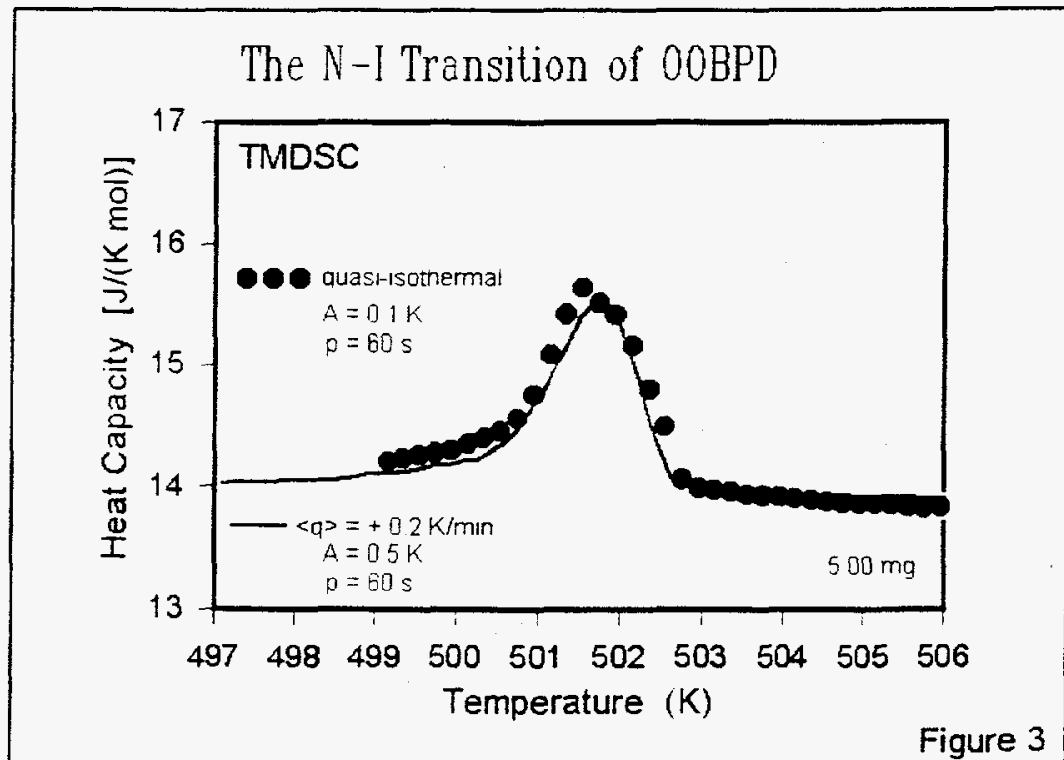




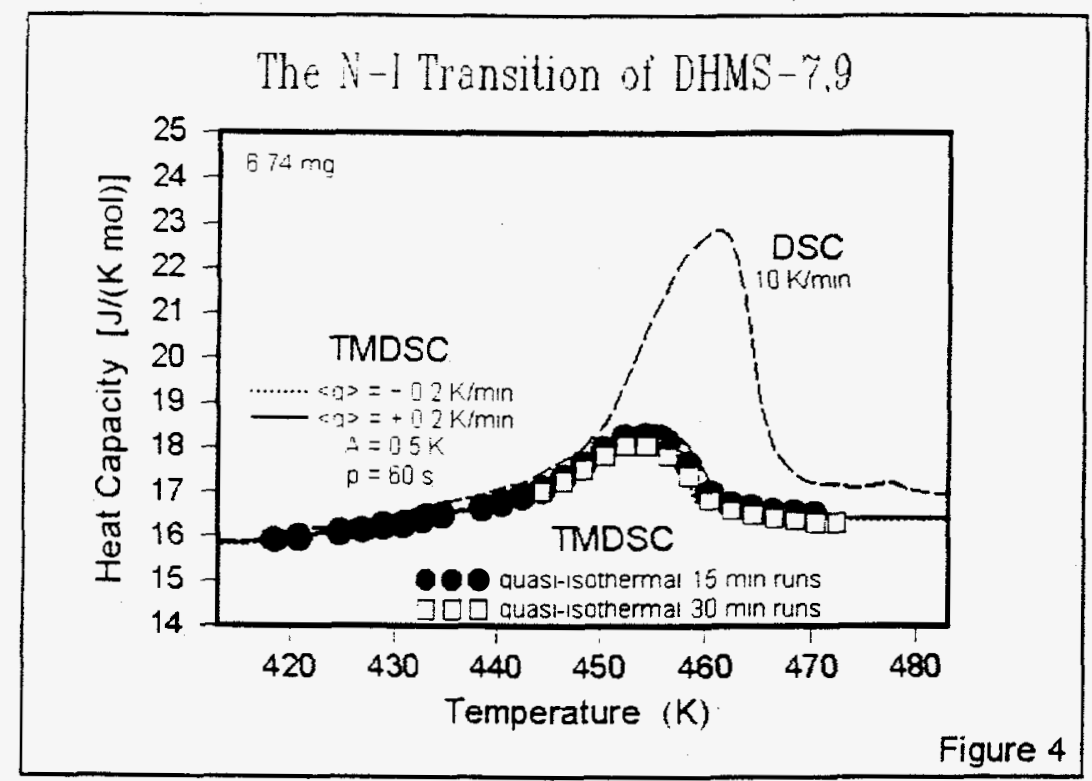

changing temperature difference between sample and sensor (sample thermocouple). Its magnitude is identical to that found with the same DSC for indium [15]. No supercooling is observed in Fig. 1. showing that no nucleation of the nematic phase is necessary. Crystallization of In. in contrast. needs a supercooling of $1.0 \mathrm{~K}$ [15]. For the different instruments of Fig. 1. the slopes are similar. The small changes of slope for heating and cooling differ for the various instruments and also for different measuring conditions with the same instrument (cooling device. $N_{2}$-flow. etc.) [15].

Bigger deviations from Eq. (3) near zero heating rate are common for all instruments. This was first discovered on calibration with In [15]. The cause seems to be that the transition starts at the bottom of the sample, close to the sensor. and fixes the sample temperature. The heat flow is governed. however. by the changing heater temperature to attain a tixed $q$, regardless of the sample temperature. As a result. the (small) temperature difference between sample and sensor changes. This changed temperature difference sets up a small. additional heat flow between sample and sensor. resulting in the lower than expected temperature of Eq. (3) on heating, and a higher on cooling. For larger q. this effect becomes negligible. There is not sufficient time to hold the bottom sample laver at the constant transition temperature. Obviously this temperature deviation around zero scanning rate is dependent on the sample mass. placement and geometry of the pan. as well as the overall DSC construction and running conditions. In the present cases the maximum effects are about $\pm 0.3 \mathrm{~K} . \pm 0.1 \mathrm{~K}$. and $\pm 0.05 \mathrm{~K}$ for the TA Instruments. Mettler-Toledo and Perkin-Elmer DSC. respectively.

The details of the $N=I$ transition are revealed by TMDSC. Figure 2 shows with the quasi-isothermal results (circles) that the heat capacity of the 4.4'azoxyanisole gradually increases on approaching the transition temperature from the 
low-temperature side. as is common for LCs [1]. A larger upturn. that may be considered a pre-transition effect. starts at $\approx 407 \mathrm{~K}$. Close to the onset of isotropization measured with traditional DSC [407.93 K. see Figs. 1 and 2 and Eq. (3)]. a large jump occurs in the quasi-isothermal TMDSC. as expected for a sharp first-order transition. The occurrence of the transition can be illustrated more clearly in the time domain. using the Lissajous traces shown in Fig. 5 (modulated heat flow $v s$. temperature plots). Starting from the left. the second set of Lissajous traces indicates that the major part of the transition occurs between 408.05 and $408.25 \mathrm{~K}$. The initial onsets of endotherms and exotherms are at $\approx 408.15 \mathrm{~K}$. The small difference from Eq. (3) can easily be accounted for by the uncertainty of the sample temperature at low q. The third set of Lissajous traces indicates some residual $N \sharp I$ transition in the sample. It is interesting to note that the initial sharp transition peaks disappear with time. reaching a new steady state of temperature gradients (compare to Fig. 6. for standard TMDSC).

Taking the beginning of the transition at the stronger upturn of heat capacity at $407 \mathrm{~K}$ and the end at $408.5 \mathrm{~K}$. as suggested by Figs. 2 and 5. the actual transition has a temperature range of about $1.5 \mathrm{~K}$. even though the main transition is much narrower $(<0.2 \mathrm{~K})$. The reversible. apparent heat capacity of the standard TMDSC is much broader when compared to the quasi-isothermal measurement. The reason for this peak broadening is the larger modulation amplitude $(\mathrm{A}=0.5 \mathrm{~K})$ and the multiple averaging used in the evaluation of the reversing heat-flow amplitude [5] $(3 / 2$ cycles $= \pm 0.3 \mathrm{~K})$. With an underlying cooling rate (dotted curve of Fig. 2) practically identical curves to heating result. supporting the fact that the transition is an equilibrium transition. The small shift is in agreement with Eq. (3).

From the reversing heat capacity of the standard TMDSC in Fig. 2 (solid and doted lines) one may be tempted to evaluate a heat of transition. It must be pointed

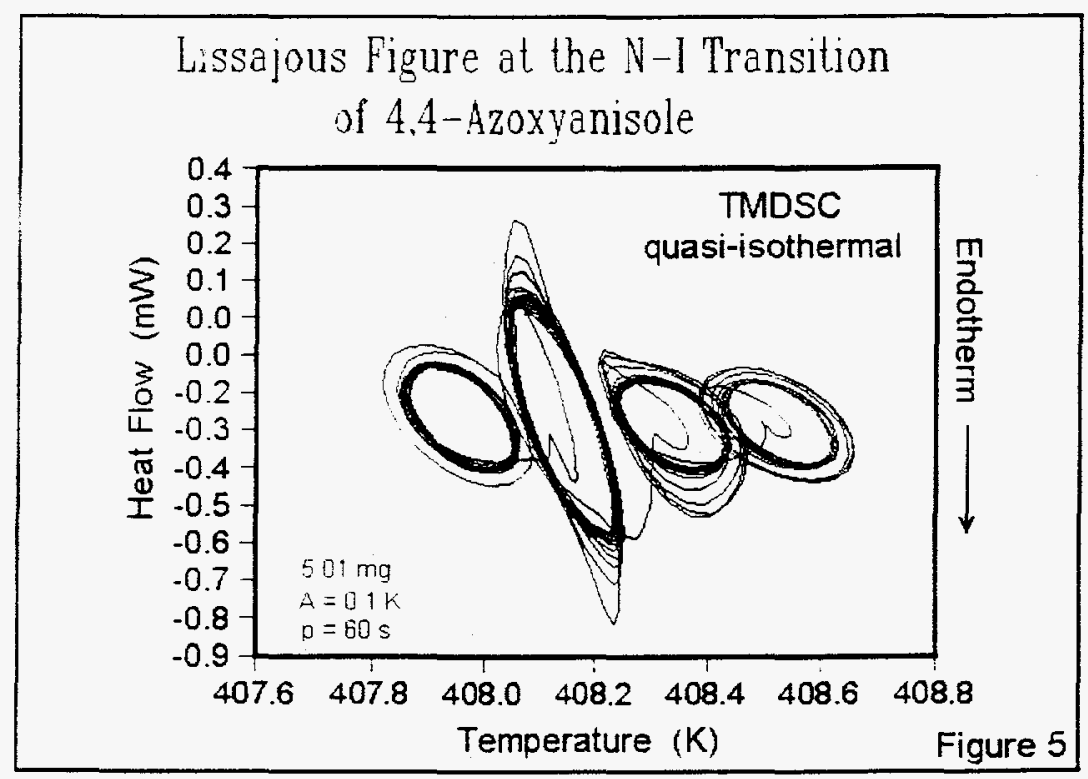


out. however. that the reversible heat flow represents only the first harmonic of a Fourier series used to fit the time-dependent heat flow [5.6]. As shown in Fig.6. the heat flow signal in the transition range (dashed line) no longer follows a sinusoidal pattern. the line shape becomes sharper and shifts its peaks from cycle to cycle as the underlying temperature increases. Therefore. in the transition range. higher harmonics of the Fourier series need to be added to describe the heat flow fully. One can. however. carry out the integration of the heat flow due to the transition in the time domain. From the plot of heat flow vs. time in Fig.6. at high and low temperatures one can establish a base line in the transition range (solid line in Fig. 6). The difference between the actual heat flow and the base line can then be related to the heats of transition. The heat of isotropization of the nematic phase for 4.4'Azoxyanisole determined by this method is $2.56 \mathrm{~J} \mathrm{~g} \mathrm{',} \mathrm{which} \mathrm{agrees} \mathrm{well} \mathrm{with} \mathrm{the}$ value obtained from traditional DSC $\left(2.52 \mathrm{~J} \mathrm{~g}^{-1}\right)$.

For OOBPD. shown in Fig. 3. only quasi-isothermal and standard TMDSC on heating were performed. Both measurements coincide and indicate that isotropization of the nematic phase of OOBPD occurs more gradually, covering a temperature range of $\approx 3.0 \mathrm{~K}$. The identical data for both measurement methods are due to the fact that the modulation amplitudes $(\mathrm{A}=0.1$ and $0.5 \mathrm{~K}$. respectively) are much smaller than the intrinsic transition width $(3.0 \mathrm{~K})$ so that the peak broadening effects. seen in the case of 4.4'-Azoxyanisole. do not occur. Since the sample is known from NMR to be relatively pure [12], the molecules must actually have a continuous series of states of intermediate order.

For the polymer sample DHMS-7,9 (Fig. 4), the breadth of the $N-I$ transition is $\approx 20 \mathrm{~K}$. The most likely explanation rests with the polydispersity of the macromolecule and local variation in chemical structure of the copolymer. i.e.. the different molecules or segments have transitions at different temperatures. It is reported in the

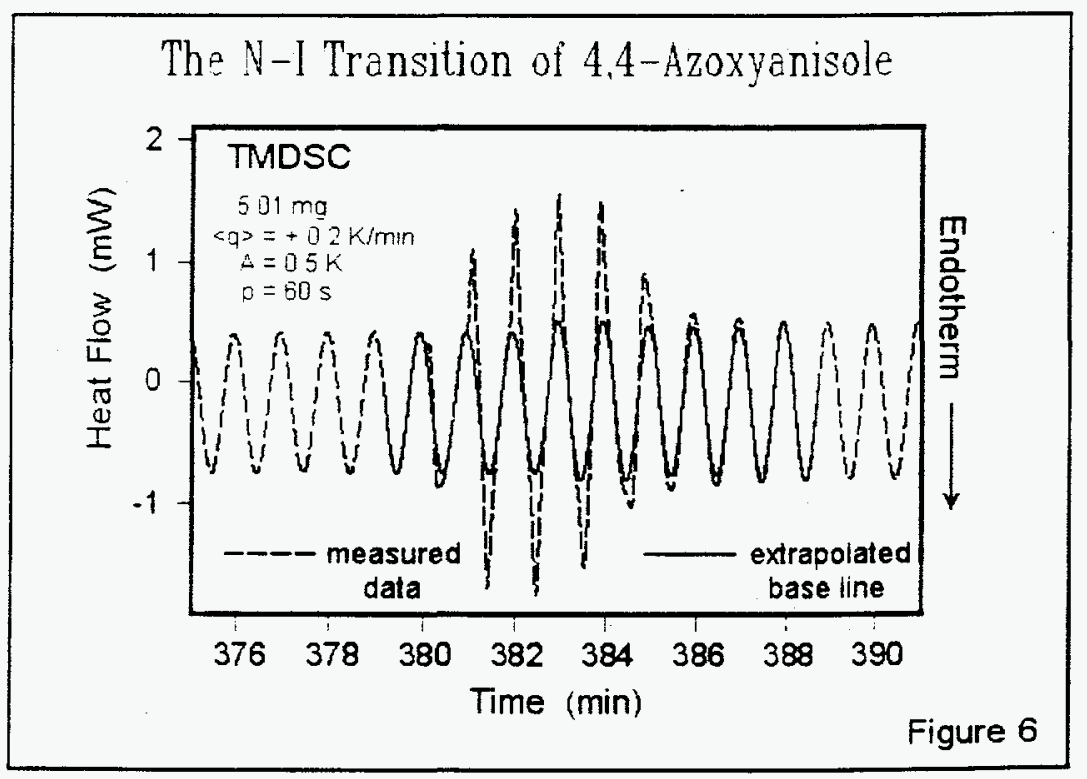


literature and seen by optical microscopy in our laboratory that at the $\mathrm{N} \rightarrow[$ transition there is a temperature interval where the nematic and isotropic phases coexist.[13].

Finally. it should be pointed out that . like in the case of 4.4'-Azoxyanisole, an "excess" heat-capacity increase below the onset isotropization temperature was also observed for OOBPD and DHMS-7.9 (see Figs. 3 and 4). This excess heat capacity is very much less above the $N \sharp I$ transition. It seems to lead to the conclusion that a certain "pre-transition" occurs in the nematic phase. Detailed investigations of this pre-transition that occurs also before melting is in progress.

\section{Acknowledgments}

This work was supported by the Div. of Materials Res.. National Science Foundation. Polymers Program. Grant \# DMR-9703692 and the Div. of Materials Sci., Office of Basic Energy Sciences. U.S. Department of Energy at Oak Ridge National Laboratory, managed by Lockheed Martin Energy Research Corporation for the U.S. Department of Energy, under contract number DE-AC05-96OR22464.

\section{References:}

1. B. Wunderlich. J. Grebowicz. Adv. Polymer Sci.. 60/61 (1984) 1.

2. J. D. Menczel and T. M. Leslie. Thermochimica Acta. 166 (1990) 309.

3. G. W. H. Höhne. H. K. Cammenga. W. Eysel. E. Gmelin and W. Hemminger, Thermochimica Acta. 160 (1990) 1.

4. A. Boller. Y. Jin and B. Wunderlich. J. Thermal Analysis. 42 (1994) 307.

5. B. Wunderlich. Y Jin and A. Boller. Thermal Acta. 238. (1994) 277.

6. B. Wunderlich. J Thermal Analysis 48, (1997) 207.

7. B. Wunderlich. A. Boller. I. Okazaki and S. Kreitmeier. Thermochim. Acta, 282/283 (1996) 143.

8. Wunderlich. “Temperature-Modulated Calorimetry." 1997. A computer assisted lecture course that is available from our World-Wide-Web site. URL: http//funnelweb. utcc.utk.edu/ athas

9. B. Wunderlich. “Thermal Analysis," Academic Press. 1990.

10. G. W. Gray. J. B. Hartley, A. Ibbotson and B. Jones. J. Chem. Soc.. (1995) 4359.

11. J. Cheng, W. Chen. Y. Jin and B. Wunderlich. Mol. Cryst. Liq. Cryst.. 241 (1994) 314.

12. J. Cheng. Y. Jin. G. Liang. B. Wunderlich. H. G. Wiedemann. Mol. Cryst. Liq. Cryst.. 213 (1992) 237.

13. J. R. Gilmore. R. H. Colby, E. Hall and C. K. Ober. J. Rheol. 38(5) (1994) 1623.

14. E. Hall. C. K. Ober, E. J. Kramer, R. H. Colby and J. R. Gilmore, Macromolecules. 26 (1993) 3764.

15. K. Ishikirivama. A. Boller, B. Wunderlich. J. Thermal Anal. 50 (1997) xxx. 